University of Wollongong

Research Online

Faculty of Engineering and Information

Faculty of Engineering and Information

Sciences - Papers: Part A

Sciences

$1-1-2014$

\title{
A multifeature-based approach for islanding detection of DG in the subcritical region of vector surge relays
}

Mollah R. Alam

University of Wollongong, mra497@uowmail.edu.au

Kashem M. Muttaqi

University of Wollongong, kashem@uow.edu.au

Abdesselam Bouzerdoum

University of Wollongong, bouzer@uow.edu.au

Follow this and additional works at: https://ro.uow.edu.au/eispapers

Part of the Engineering Commons, and the Science and Technology Studies Commons

Research Online is the open access institutional repository for the University of Wollongong. For further information contact the UOW Library: research-pubs@uow.edu.au 


\title{
A multifeature-based approach for islanding detection of DG in the subcritical region of vector surge relays
}

\begin{abstract}
Anti-islanding protection is an important requirement which has to be considered prior to the integration of distributed generation into electricity grids. Conventional vector surge (VS) relays are usually used to detect islanding; however, there is a nondetection zone (NDZ) wherein islanding incidents are undetectable by VS relays. This paper proposes a multifeature-based technique for islanding detection in the subcritical region, defined as a subregion of the NDZ. In the proposed method, features are extracted from five network variables. The extracted features are then used as inputs to a support vector machine to classify the event as islanding or nonislanding. A test network was used to generate a large number of islanding and nonislanding events with different load types. The proposed method is tested with the most critical islanding cases associated with NDZ of VS relays. Furthermore, all possible combinations of deficit and excess of active and reactive power imbalance, which may exist during the occurrence of an island, are considered in the testing phase. Experimental results demonstrate that the proposed method can successfully detect islanding events in the subcritical region, where a VS relay is expected to fail.
\end{abstract}

\section{Keywords}

approach, multifeature, region, subcritical, surge, dg, detection, vector, islanding, relays

\author{
Disciplines \\ Engineering | Science and Technology Studies
}

\section{Publication Details}

M. R. Alam, K. M. Muttaqi \& A. Bouzerdoum, "A multifeature-based approach for islanding detection of DG in the subcritical region of vector surge relays," IEEE Transactions on Power Delivery, vol. 29, (5) pp.

2349-2358, 2014. 


\title{
A Multifeature-Based Approach for Islanding Detection of DG in the Subcritical Region of Vector Surge Relays
}

\author{
M. R. Alam, Student Member, IEEE, K. M. Muttaqi, Senior Member, IEEE, and A. Bouzerdoum, \\ Senior Member, IEEE
}

\begin{abstract}
Anti-islanding protection is an important requirement which has to be considered prior to the integration of distributed generation into electricity grids. Conventional vector surge (VS) relays are usually used to detect islanding; however, there is a nondetection zone (NDZ) wherein islanding incidents are undetectable by VS relays. This paper proposes a multifeature-based technique for islanding detection in the subcritical region, defined as a sub-region of the NDZ. In the proposed method, features are extracted from five network variables. The extracted features are then used as inputs to a support vector machine to classify the event as islanding or nonislanding. A test network was used to generate a large number of islanding and nonislanding events with different load types. The proposed method is tested with the most critical islanding cases associated with NDZ of VS relays. Furthermore, all possible combinations of deficit and excess of active and reactive power imbalance, which may exist during the occurrence of an island, are considered in the testing phase. Experimental results demonstrate that the proposed method can successfully detect islanding events in the subcritical region, where a VS relay is expected to fail.
\end{abstract}

Index Terms--Distributed generation (DG), islanding detection, nondetection zone (NDZ), support vector machine (SVM), vector surge (VS) relay.

\section{INTRODUCTION}

$\mathrm{D}$ ISTRIBUTED Generation (DG) has been receiving special attention over the past few years, due to its ability to improve voltage profiles and power quality (PQ), and to reduce power losses in distribution systems [1]. However, integration of DG sources in a grid-connected environment gives rise to several operational issues. One such issue is islanding, which can be defined as a condition when a segment of the utility network with DG is disconnected from the main supply, but the DG in the isolated portion remains active and generates power [2]. Islanding or loss of mains (LOM) event needs to be detected accurately in order to avoid probable hazardous conditions. Unintentional islanding is a concern and, therefore, the normal practice is to disconnect all distributed resources (DRs) immediately after the onset of islanding. The IEEE 1547-2003 standard recommends the

Mr. M. R. Alam, A/Prof. K. M. Muttaqi, and Prof. A. Bouzerdoum are with the Australian Power Quality and Reliability Center, School of Electrical, Computer and Telecommunications Engineering, University of Wollongong, NSW 2522, Australia (Emails: mra497@uowmail.edu.au; kashem@uow.edu.au; a.bouzerdoum@uow.edu.au). disconnection time to be less than 2 seconds; and islanding detection relays are used for this purpose. Different techniques are used in islanding detection schemes, and they include active, passive and remote techniques. In active detection methods, a small disturbance signal is introduced into the system and a feedback technique or control mechanism is employed to detect the changes in the frequency or voltage when islanding occurs [3]. This method provides several advantages as it tends to have fast response with small nondetection zone (NDZ); however, using this method leads to degradation in PQ due to the perturbation imposed on the system. Recently, active islanding detection techniques, such as slip-mode frequency shift [4], active frequency drift [5], Sandia frequency and voltage shift method [6], have been reported. Remote methods are communication-based techniques; they are more effective, accurate and robust, but incur high cost. Passive methods use different system parameters, for example, voltage and frequency, and compare them to a prespecified threshold to decide the occurrence of islanding. Passive methods do not affect the normal operation of the DG system and are easy to implement, but they lack in accuracy.

Due to the trade-off between accuracy and cost, passive methods such as rate-of-change-of-frequency (ROCOF) and vector surge (VS) relays are normally used for islanding detection. However, according to the performance curve of ROCOF relays given in [7], the overall performance deteriorates when the power imbalance falls below a certain specified limit. Similarly, for a particular relay setting and detection time, VS relays suffer from the limitation of nondetection zone (NDZ) [7].

In recent years, several computational intelligence based passive techniques have been proposed for islanding detection [2], [8]. The fuzzy rule-based classifier, decision tree (DT) approach and wavelet-based technique have attracted widespread attention [9]-[11]. Support vector machines (SVMs) are another type of classification methods [12], [13], which have been widely used in many applications [14]. In [3], islanding detection of inverter-based DG was evaluated using seven features and four classifiers: Decision Trees, Radial Basis Functions, SVMs and Probabilistic Neural Networks. In [15], the authors carried out a task of islanding detection in the presence of three types of DG units: inverter-interfaced DG, synchronous-type DG, and multiple DG units (synchronous type and/or inverter interfaced DG). In their approach, 21 features are first extracted, from which four features are selected as input to a Random Forest (RF) classifier. By contrast, this 
paper proposes a technique for islanding detection inside the NDZ of VS relays. Five network variables are used for feature extraction; they include voltage, frequency, phase angle of voltage, rate of change of frequency, and rate of change of voltage (ROCOV). Although the proposed method works for islanding cases outside the NDZ of VS relays, it mainly aims for islanding events inside the subcritical region (SCR), which is a sub-region of NDZ of VS relays. In [3] and [15], islanding events with different types of loads were not highlighted, whereas in this paper, islanding events in the presence of constant impedance, constant current and constant power loads are investigated. Moreover, combination of deficit and excess of active and reactive power imbalance inside the islanded segment makes the islanding detection even more complex [7]. In this work, islanding events, considering all possible combinations of active and reactive power imbalance (deficit and excess) under constant impedance, constant current and constant power loads, are investigated and detected with a high degree of accuracy.

The remainder of this paper is organized as follows. Section II defines the subcritical region of VS relays in the presence of synchronous generator-based DG; the influence of load types on the boundaries of SCR is also investigated. Section III presents the proposed method describing the procedure of feature extraction, event classification using SVM, and islanding detection. Section IV presents the experimental evaluation of the proposed method. Section V concludes this paper.

\section{SubCRITICAL REgION OF VS RELAYS}

For VS relays, the critical power imbalance is the active power imbalance for which the relay can marginally detect islanding; for any smaller value, it is unable to perform within a permissible timeframe. The zone below the critical power imbalance is referred to as the NDZ [16]. We define the Subcritical Region as the sub-region of NDZ wherein the proposed method can successfully detect islanding events; it extends from the critical power imbalance to a lower limit determined by the load type of the system.

Critical active power imbalance $\left(\Delta P_{v s_{-} c r i t}\right)$ acts as a separation boundary between the detection and nondetection zone of VS relays. This separation boundary also indicates the boundary limit of SCR. Therefore, the formulation of the boundary limit of SCR is carried out by using the concept of critical active power imbalance; for more details, see Section II-A. Moreover, in Sections II-B and II-C, the impacts of load dynamics and reactive power imbalance are highlighted to determine the pessimistic boundary limit of SCR.

\section{A. Formulation of Boundary Limit of SCR for VS Relay}

Fig. 1 illustrates a network with a synchronous generator (SG)-type DG operating in a grid-connected mode. During islanding, the load is fed from the DG source only. Therefore, the boundary of the SCR of the VS relay can be determined by analyzing the operation of the SG. The dynamic behavior of the synchronous generator can be characterized using the machine swing equation [17]:

$$
\frac{2 H}{\omega_{0}} \frac{d^{2} \delta}{d t^{2}}=P_{m}-P_{l}=-P_{S}=\Delta P
$$

where $H$ is the generator inertia constant, $\omega_{0}$ is the synchronous speed, and $\delta$ is the rotor angle with respect to the synchronously rotating reference frame. Since $\delta$ varies with time during the transient period of islanding, the rotor speed $\omega_{r}$ can be presented as:

$$
\omega_{r}=\omega_{0}+\frac{d \delta}{d t}
$$

Assuming $t_{0}$ is the onset of islanding, at $t=t_{0}, \omega_{r}=\omega_{0}$ and hence, $d \delta / d t=0$. Taking this initial condition into consideration and integrating equation (1) twice, yields the rotor angle $\delta$ at time $t$ :

$$
\delta(t)=\delta_{0}+\frac{\omega_{0} \Delta P}{4 H}\left(t-t_{0}\right)^{2}
$$

where $\delta_{0}$ is the rotor angle at steady state prior to islanding.

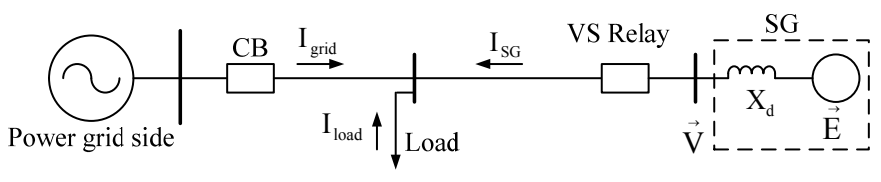

Fig. 1. A synchronous type DG operating in a grid-connected mode.

For the VS relay, the difference of $\delta$ within each cycle interval is measured and compared with VS relay setting $\alpha$. The VS relay is triggered when the difference between rotor angles $(\Delta \delta)$ within any of the cycle intervals crosses the relay setting $\alpha$. Figure 2 illustrates an islanding situation where the variation of the terminal voltage $v(t)$ is shown in Fig. 2(a), and the rotor angle $\delta(t)$ is presented in Fig. 2(b). A moving window of fixed width $\Delta T$ seconds in duration is proposed to calculate the change in rotor angle.

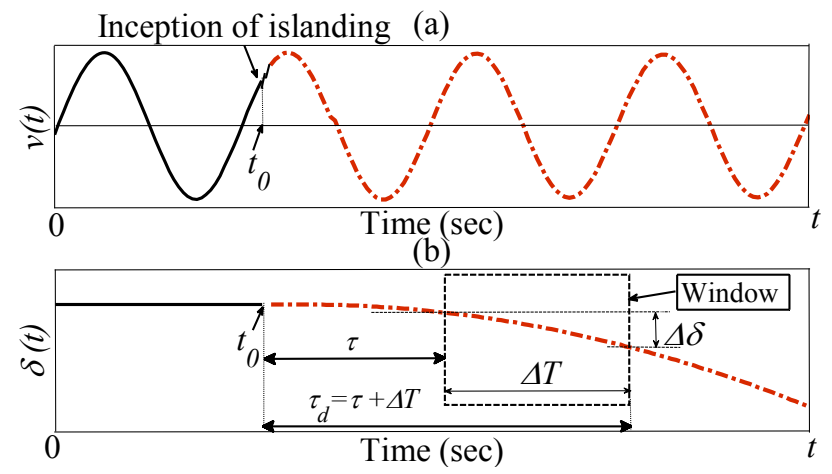

Fig. 2. Pre-islanding (solid line) and post-islanding (dash-dot line): (a) Terminal voltage $v(t)$ at SG (Synchronous Generator) end (b) rotor angle $\delta(t)$ with moving window to detect rotor angle change.

Let $\Delta \delta(t)$ denote the rotor angle change between $t$ and $(t+\Delta T)$. Using (3), the rotor angle change is obtained as:

$$
\Delta \delta(t+\Delta T)=\delta(t+\Delta T)-\delta(t)=\frac{\omega_{0} \Delta P}{4 H}\left(2 \tau \Delta T+\Delta T^{2}\right)
$$

where $\tau=t-t_{0}$. An islanding event is detected if $\Delta \delta(t)$ exceeds the relay setting $\alpha$, which gives 


$$
\frac{\omega_{0} \Delta P}{4 H}\left(2 \tau \Delta T+\Delta T^{2}\right) \leq \alpha
$$

where $\Delta P$ is negative considering load is higher than generation during islanding, and $\alpha$ is negative due to proposed moving window based method of detecting $\Delta \delta$ with negative $\Delta P$. Using the expression of total detection time $\tau_{d}\left(\tau_{d}=\tau+\Delta T\right)$, expression (5) can be re-written as:

$$
\frac{\omega_{0} \Delta P}{4 H}\left(2 \tau_{d} \Delta T-\Delta T^{2}\right) \leq \alpha
$$

Taking the equality constraint of (6) and setting $\Delta T=0.02 \mathrm{sec}$ (i.e. one cycle in $50 \mathrm{~Hz}$ system), Fig. 3 (i.e. $\tau_{d}$ versus $\Delta P$ ) is obtained for a specific relay setting. For an allowable detection time $\left(\tau_{d}\right)$, the critical power $\Delta P_{v s_{-} c r i t}$, which separates detection and nondetection zone, is indicated by the vertical dotted line.

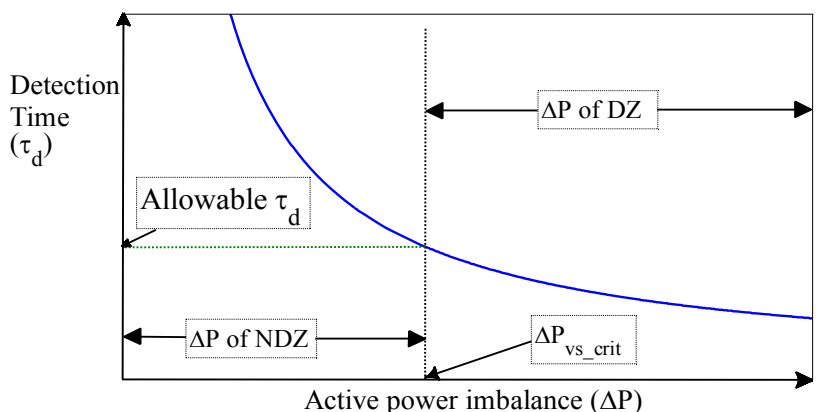

Fig. 3. Islanding detection time of VS relay as a function of $\Delta P$; and $\Delta P_{v s_{-} \text {crit }}$ or boundary limit of SCR separating detection zone (DZ) and NDZ.

To find the minimum absolute value of $\Delta P$, i.e. critical active power imbalance $\left(\Delta P_{v s_{-} \text {crit }}\right)$ of VS relay, a window width $(\Delta T)$ of 0.02 second is considered, as it is the period of fundamental frequency of $50 \mathrm{~Hz}$ voltage signal. So, by putting $\Delta T=$ $0.02 \mathrm{sec}$ and $\tau_{d}=0.2 \mathrm{sec}$ in (6), $\Delta P$ of VS relay can be obtained with specific relay setting $\alpha$. Thus, as an example, for absolute value of $\alpha=10^{\circ}$ and $H=1.5$, the minimum absolute value of $\Delta P$, i.e. $\Delta P_{v s_{-} c r i t}$ of VS relay can be obtained as 0.4386 $\mathrm{pu}$ which indicates the boundary limit of SCR.

\section{B. Impact of Load Types on Boundary Limit of SCR}

As reported in [17], taking the load dynamics and power imbalance factor into consideration, the generalized expression of active power imbalance for constant power (constant $P$ ), constant impedance (constant $Z$ ), or constant current (constant $I$ ) load can be obtained from the following:

$$
\Delta P=\Delta P_{0}^{1 / P F}
$$

where $\Delta P_{0}$ is the initial power imbalance value at the instant of islanding, $\triangle P$ denotes the final active power imbalance for constant $Z$, constant $I$ or constant $P$ load; and $P F$ is a factor which is in the range of $1.2 \geq P F \geq 0.8$ considering average power imbalance variation of $\pm 20 \%$. Empirical value of $P F$ is selected as $1,0.8$ and 0.9 for constant $P$, constant $Z$ and constant $I$ load respectively when there is a deficit of electric power [17]. Thus, by replacing $\Delta P$ given in (6) with $\Delta P_{0}^{1 / P F}$ given in (7), the absolute critical active power imbalance for constant $P$, constant $I$ and constant $Z$ load can be ob- tained as $0.4386 \mathrm{pu}, 0.4763 \mathrm{pu}$ and $0.5172 \mathrm{pu}$ respectively. Hence, boundary limit of SCR for the VS relay corresponds to the boundary when active power imbalance within the islanded network falls down to $43.86 \%, 47.63 \%$ and $51.72 \%$ for constant $P$, constant $I$ and constant $Z$ load respectively.

\section{Impact of Reactive Power Imbalance on Boundary Limit of $S C R$}

In this paper, the boundary limit of SCR is defined in terms of maximum numerical value of critical active power imbalance for the most pessimistic cases. To this end, the foll- wing four combinations or scenarios [7] are considered: (a) deficit of $\Delta \mathrm{P}$ and $\Delta \mathrm{Q}$, (b) deficit of $\Delta \mathrm{P}$ and excess of $\Delta \mathrm{Q}$, (c) excess of $\Delta \mathrm{P}$ and deficit of $\Delta \mathrm{Q}$, and (d) excess of $\Delta \mathrm{P}$ and $\Delta \mathrm{Q}$.

The impact of $\Delta \mathrm{Q}$ on the boundary limit of SCR is investigated by carrying out repeated dynamic simulation on the system shown in Fig. 4 with different ranges of active and reactive power imbalance for different load types. Then the detection time for specific relay setting (i.e. $\alpha=10^{\circ}$ ) is obtained. The power imbalance corresponding to the detection time of $200 \mathrm{~ms}$ [7] is considered as critical active power imbalance. From the simulation results, the maximum numerical value of boundary limit of SCR is obtained for the power imbalance due to the deficit of active and reactive power inside the islanded network. Therefore, the pessimistic boundary limit of SCR is defined considering the deficit of active and reactive power imbalance scenario. For this case study, it is observed that $\Delta \mathrm{Q}$ has negligible impact on the boundary limit of SCR for constant power load; $\Delta P_{v s_{-} \text {crit }}$ is obtained as $45.8 \%$ for different ranges of $\Delta \mathrm{Q}$. But the influence of $\Delta \mathrm{Q}$ on boundary limit of SCR is quite evident for constant $Z$ and constant $I$ load, as shown in Fig. 5.

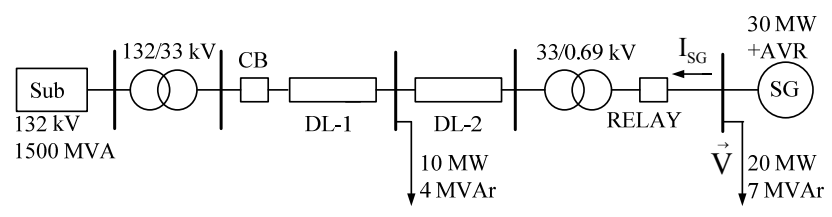

Fig. 4. Single line diagram of a simple distribution system [16].

From Fig. 5, it can be observed that the pessimistic cases are obtained by keeping the reactive power imbalance at its maximum. In this study, a reactive power imbalance of $50 \%$ has been considered as the maximum reactive power imbalance, since during electric system islanding the system voltage may collapse whenever the reactive power imbalance exceeds $50 \%$ [18]. Thus, for VS relay $\left(\alpha=10^{\circ}\right.$ and detection time $=$ $200 \mathrm{~ms}$ ), the boundary limit of pessimistic SCR is indicated by the active power imbalance level of $66 \%, 58 \%$ and $45.8 \%$ for constant impedance, constant current and constant power load respectively. It should be noted that a deficit of active and reactive power imbalance scenario is considered for generating the data of Fig. 5. 

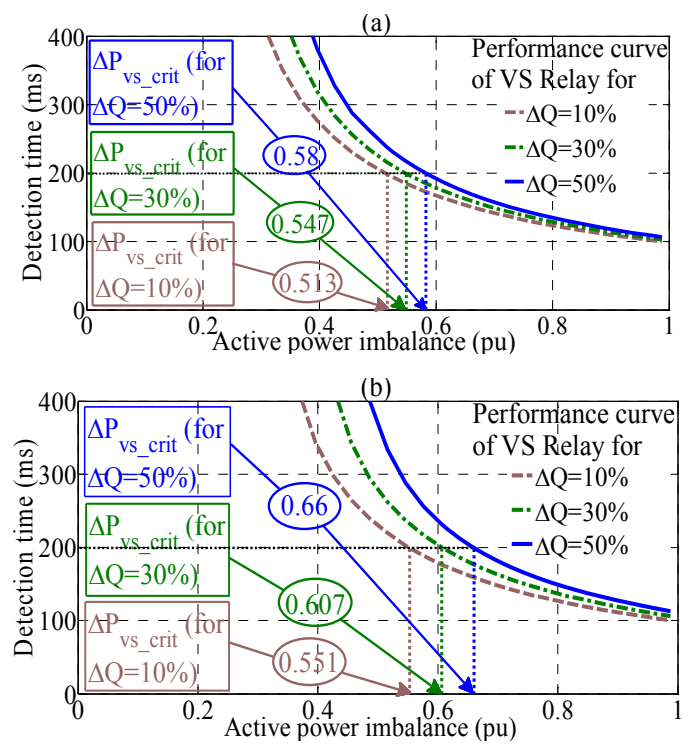

Fig. 5. Impact of $\Delta \mathrm{Q}$ on $\Delta P_{v s_{-} \text {crit }}$ or boundary limit of SCR of VS relay (relay setting: $\alpha=10^{\circ}$ ) for (a) constant current and (b) constant impedance load.

In the remaining sections, the proposed multifeature-based approach is discussed and the developed method is tested inside the boundary limit of pessimistic SCR.

\section{MultifeAture-BASED IsLANDING DeteCtion}

The proposed method is described in the following three sub-sections. Sub-section $A$ describes the process of extraction of network variables and features associated with islanding and nonislanding events. Sub-section $B$ narrates the theory of SVM (Support vector machine) pertinent to the classification of two groups of data. The procedure of detection of islanding is presented in sub-section $C$.

\section{A. Extraction of Network Variables and Features}

In the proposed method, five variables are used for feature extraction: normalized frequency $f$, rate of change of frequency $\rho_{f}=d f / d t$, normalized phase angle of voltage $\theta$, normalized voltage $V$, and rate of change of voltage $\rho_{v}=$ $d V / d t$. These var- iables are extracted from the terminal voltage of the DG. The frequency variable, $f$, is obtained by taking the time-inverse of the duration of two consecutive rising zero-crossing points of voltage signal. $\operatorname{ROCOV}\left(\rho_{v}\right)$ and ROCOF $\left(\rho_{f}\right)$ are extracted from the change of voltage and frequency within small time interval $\Delta t$ (where $\Delta t$ can be typically $5 \times 10^{-4} \mathrm{~s}$ ). The process of extraction of phase angle $(\theta)$ of $V$ is shown in Fig. 6.

In order to obtain the phase angle $(\theta)$ from the terminal voltage $v(t)$, the synchronously rotating reference frame is represented by a reference signal $s(t)$ with $50 \mathrm{~Hz}$ frequency. Since, detection time of $200 \mathrm{~ms}$ is considered, $s(t)$ of 10 cycles is shown in Fig. $6(\mathrm{a})$, and $v(t)$ of 10 cycles duration is processed to obtain the phase angle deviation from reference signal $s(t)$ as shown in Fig. 6 (b). As indicated in Fig. 6 (b), four points: two zero-crossing points, one positive half-cycle peak and another negative half-cycle peak points, are determined in each cycle. This procedure is followed to obtain $m+1$ number of points during the duration of 10 cycles of $v(t)$ signal; the points are denoted as $t_{0}, t_{1}, t_{2}, \ldots . t_{m}$. Then $\theta(m)$, as shown in Fig. 6(c), is obtained by using the following expression:

$$
\theta(m)=\left[m \times \frac{T}{4}-\left(t_{m}-t_{0}\right)\right] \times \frac{360}{T} \text { for } m=0,1,2, . .
$$

where $\theta(m)=$ phase angle of $v(t)$ in degrees with respect to $s(t)$, and $T=$ time period of $s(t)$.
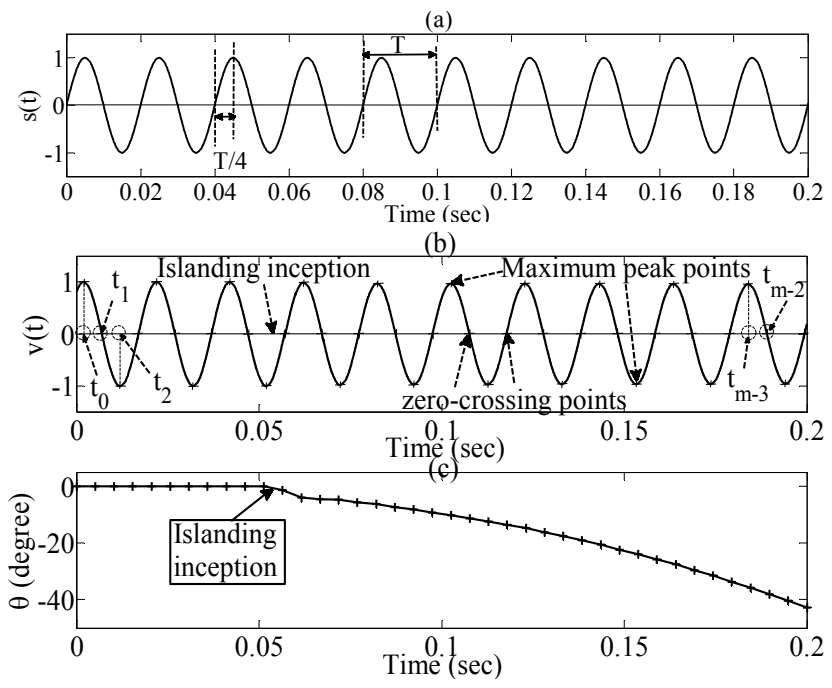

Fig. 6. Extraction of phase angle $(\theta)$ : (a) Reference signal $s(t)$, (b) Terminal voltage $v(t)$, and (c) Phase angle of $v(t)$ with respect to $s(t)$.

In Fig. 7, the normalized five variables are highlighted within a typical window block of 10 cycles to extract five features. These features are obtained by taking the standard deviation (SD) of all samples inside the window block for each of the five variables separately. Thus, five features are used as inputs to a SVM classifier, which is discussed in the following sub-sections.
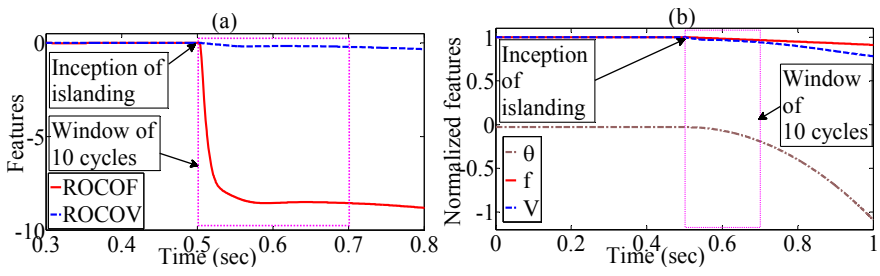

Fig. 7. Normalized network variables: (a) ROCOF and ROCOV, and (b) $\theta, f$ and $V$ under islanding condition (islanding inception at $0.5 \mathrm{sec}$ ).

\section{B. Classification of Events using SVM}

In the proposed method, (binary) SVM is applied to classify two groups of data: islanding and nonislanding. Therefore, theory of SVM is briefly discussed below.

Given a training set $\mathcal{X}=\left\{\left(\boldsymbol{x}_{1}, y_{1}\right),\left(\boldsymbol{x}_{2}, y_{2}\right), \ldots \ldots,\left(\boldsymbol{x}_{n}, y_{n}\right)\right\}$, where $\boldsymbol{x}_{n}$ represents a real valued $d$-dimensional input or feature vector $\left(\boldsymbol{x}_{n} \in \mathfrak{R}^{d}\right)$; and $y_{n}=\{-1,+1\}$ is a label indicating the class of $\boldsymbol{x}_{n}$ or the desired output. The main objective of (binary) SVM is to separate these two classes of features by introducing a decision boundary hyperplane determined by an orthogonal weight vector $\boldsymbol{w}$ and a bias $b$. The hyperplane can 
be presented as:

$$
g(\boldsymbol{x})=\boldsymbol{w}^{T} \boldsymbol{x}+b
$$

The function $\operatorname{sgn}(g(x))$ can be used as decision function and it gives the label $\{-1,+1\}$ indicating the class of input feature vector $\boldsymbol{x}$.

To deal with linearly non-separable training sets, soft-margin support vector machines are considered. In soft margin SVMs, the weight vector $\boldsymbol{w}$ and the bias term $b$ are determined by solving the following problem [12], [19]:

$$
\begin{array}{lll}
\underset{w, \xi}{\operatorname{minimize}} & \frac{1}{2}\|\boldsymbol{w}\|^{2}+C \sum_{n=1}^{N} \xi_{n} \\
\text { subject to } & y_{n}\left(\boldsymbol{w}^{T} \boldsymbol{x}_{n}+b\right) \geq 1-\xi_{n} & \text { for } n=1,2, \ldots \ldots N \\
& \xi^{\prime} \geq 0 & \text { for } n=1,2, \ldots . . N
\end{array}
$$

where the slack variable $\xi_{n}$ measures the distance between the margin and the training samples lying on the wrong side of the margin. The parameter $C$ in (10) is called a regularization parameter and it determines the trade-off between the maximization of the margin and the minimization of classification error.

Lagrange multipliers $\beta_{n} \geq 0$ and $\gamma_{n} \geq 0$ are introduced to solve the constrained QP (Quadratic programming) problem presented in (10) and (11). Hence, the Lagrange functional can be shown as [19]:

$$
\begin{aligned}
& Q(\boldsymbol{w}, b, \boldsymbol{\xi}, \boldsymbol{\beta}, \boldsymbol{\gamma}) \\
& =\frac{1}{2}\|\boldsymbol{w}\|^{2}+C \sum_{n=1}^{N} \xi_{n}-\sum_{n=1}^{N} \beta_{n}\left\{y_{n}\left(\boldsymbol{w}^{T} \boldsymbol{x}_{n}+b\right)-1+\xi_{n}\right\}-\sum_{n=1}^{N} \xi_{n} \gamma_{n}
\end{aligned}
$$

Expression (12) is solved by introducing the dual formulation of the problem which is as follows [19]:

$$
\underset{\beta}{\operatorname{maximize}}\left\{\sum_{n=1}^{N} \beta_{n}-\frac{1}{2} \sum_{n=1}^{N} \sum_{m=1}^{N} \beta_{n} \beta_{m} y_{n} y_{m} \boldsymbol{x}_{n}^{T} \boldsymbol{x}_{m}\right\}
$$

subject to $\sum_{n=1}^{N} \beta_{n} y_{n}=0$ and $C \geq \beta_{n} \geq 0$ for $n=1,2, \ldots . N$

The solution of the dual problem of (13) and (14) yields $\boldsymbol{w}$ as below:

$$
\boldsymbol{w}=\sum_{n=1}^{N} \beta_{n} y_{n} \boldsymbol{x}_{n}
$$

The $\boldsymbol{w}$ has an expansion in terms of a subset of the training data where the Lagrange multipliers $\beta_{n} \neq 0$. Those training data also satisfy the Karush-Kuhn-Tucker (KKT) condition

$$
\beta_{n}\left\{y_{n}\left(w^{T} x_{n}+b\right)-1+\xi_{n}\right\}=0 \text { for } n=1,2, \ldots . N
$$

According to (16), the training vectors corresponding to nonzero Lagrange multipliers are needed to describe the hyperplane and these training vectors are specified as support vectors (SVs). Hence, the decision boundary $g(x)$ is determined by the SVs only:

$$
g(\boldsymbol{x})=\sum_{\beta_{n}>0} \beta_{n} y_{n} \boldsymbol{x}_{n}^{T} \boldsymbol{x}+b
$$

where $\boldsymbol{x}$ is the input test vector, $\boldsymbol{x}_{n}^{T} \boldsymbol{x}$ is the inner product, $b$ is the bias term and the condition $\beta_{n}>0$ allows only the SVs of the data set.

For linearly non-separable classes, the original input space is mapped into a high-dimensional dot product space, known as feature space [12], [13]. To avoid the cumbersome task of visiting the high-dimensional feature space, a trick of kernel function is applied. Substituting for the inner product of $\boldsymbol{x}_{n}^{T} \boldsymbol{x}_{m}$ in (13) by a kernel function $K\left(\boldsymbol{x}_{n}, \boldsymbol{x}_{m}\right)$, similar type of dual formulation problem can be obtained. Upon solving the optimization problem, we obtain the decision function

$$
g(\boldsymbol{x})=\sum_{S V \text { sor } \beta_{n}>0} \beta_{\beta} y_{n} K\left(\boldsymbol{x}_{n}, \boldsymbol{x}\right)+b
$$

Examples of popular kernels include the Radial Basis Function (RBF) kernel, $K(x, y)=\exp \left\{-\frac{|x-y|^{2}}{2 \sigma^{2}}\right\}$, and the Polynomial kernel, $K(x, y)=\left(x^{T} y+1\right)^{p}$.

\section{Detection of Islanding}

In the proposed method, an SVM classifier is first trained off-line by taking the input features associated with all possible scenarios of islanding and nonislanding events which may exist in DG networks. To do so, several islanding and nonislanding events are generated and input features are extracted from any targeted DG location, as presented in Section III-A. The feature vector $\boldsymbol{x}$ comprises five input features extracted from a ten-cycle window of the voltage waveform. In our experiments, the location of islanding onset is known (ground truth); therefore, a window is considered to be an islanding case if it includes the islanding onset anywhere. We use five-fold cross-validation to determine the SVM regularization parameter $C$, the bandwidth of the RBF kernel $\sigma$, and the degree of the polynomial kernel $p$.

The flow chart presented in Fig. 8 shows the algorithm and steps of applying the trained SVM to detect islanding. Five dimensional features $\left(\boldsymbol{x}_{n}\right)$ are obtained from the available voltage signal at DG connection point. These features are tested with the trained SVM. Thus, the classification result which leads to either islanding $\left(y_{n}=-1\right)$ or nonislanding $\left(y_{n}=+1\right)$ is obtained. If islanding is detected, a trip signal is sent to disconnect the DG.

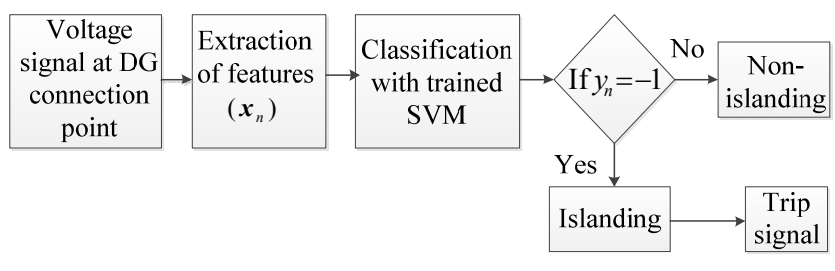

Fig. 8. Flow chart for detection of islanding.

\section{RESUlts AND Discussions}

The proposed multifeature-based islanding detection technique is evaluated with a test network containing synchronous type DG units. The description of the test system, generation 
of data and classification of events using the proposed method are discussed in the following sub-sections.

\section{A. Test Case}

A test distribution network shown in Fig. 9 is simulated in MATLAB/SIMULINK to extract the necessary features required for classification. The simulated system is a radial distribution network connected with the transmission network of Moree substation, NSW, Australia. Data of transmission network are obtained from TransGrid, a Transmission utility of New South Wales, Australia. Three 6-MVA synchronous generators (SG) are connected to the network through $33 / 0.69 \mathrm{kV}$ transformers.

The events for which a part of the network energized by DG is isolated from the grid system are considered as islanding conditions. These conditions are generated by artificially created instantaneous faults at some nodes in the transmission or distribution feeder that result in DG islanding. Normal events that may exist in practical power systems due to capacitor switching, loss of lines, load addition, load disconnection, etc., for which DG energized network is not isolated, are considered as nonislanding conditions. Thus, in the test network, the situations that might appear during islanding and nonislanding conditions are as follows:

- Tripping of main circuit breaker (CB) resulting to an islanded system with DG at the presence of different range of active and reactive power imbalance in the islanded section.

- Opening of any breakers between main grid and DG.

- Events that could trip breakers $\left(\mathrm{CB}_{1}\right.$ or $\left.\mathrm{CB}_{2}\right)$ in transmission network, and eventually island the DG under study.

- Load disconnection from the distribution network.

- Switching of capacitor bank in the network.

- Loss of line in the distribution system, apart from the line connected to the target DG.

- Occurrence of three phase fault in a distribution line, apart from the line connected to the target DG.

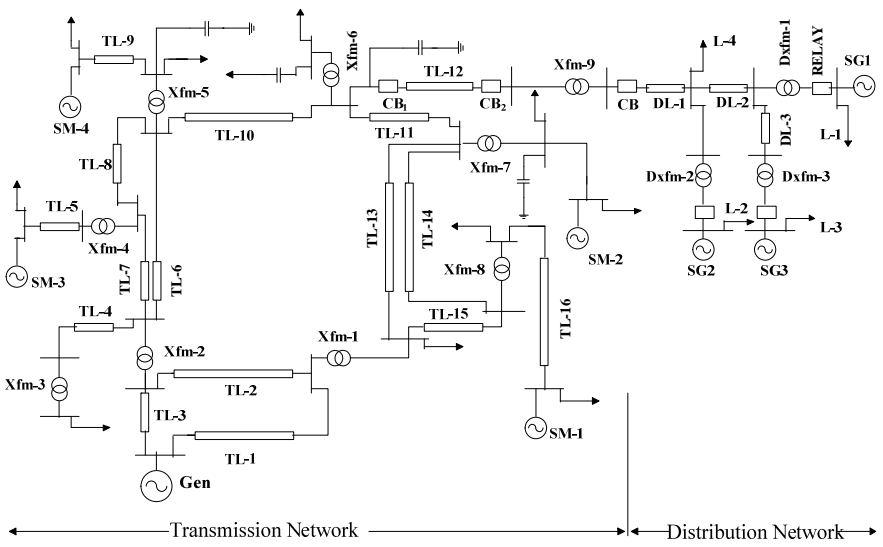

Fig. 9. Single line diagram of a test distribution network under study.

$2.0 \mathrm{kHz}$ sampling rate is used in the simulation of the test system. The relays for circuit breakers are located at the connection points of transformers of SG1, SG2 and SG3 respectively, to sense and measure the voltage signals during islanding and nonislanding conditions. Several islanding and nonislanding events are generated taking the aforementioned conditions into consideration. Three types of loads, constant impedance, constant current and constant power, are considered separately while generating the islanding and nonislanding events. Additionally, deficit and excess of active power imbalance (varied from $0 \%$ to $100 \%$ ) and reactive power imbalance (varied from $0 \%$ to $50 \%$ ) are considered when generating the different islanding events.

\section{B. Classification and Analysis of Results}

A total of 2,022 events are generated. This includes 1,302 islanding and 720 nonislanding events. The number of training and test events (including islanding and nonislanding) are presented in Table I. SVM is trained using the procedure presented in sub-section III-C. The trained SVM is tested with the 1,542 test events (consisting of 1,062 islanding and 480 nonislanding) to investigate the performance of the proposed method, especially under islanding conditions when power imbalance in the islanded network is less than the critical active power imbalance. It is to be noted that the training data was a different subset from the test data; however, both sets are taken from the same SimPower system model.

Overall, the performance of the proposed method using linear kernel SVM is shown in Table II. The pessimistic cases as mentioned in section II are also presented to show the classifier's performance inside the boundary limit of SCR. The performance of the proposed method using Gaussian RBF kernel and polynomial kernel SVM is also investigated for the special events that are associated with SCR. For this investigation, all test cases of nonislanding and islanding events inside the boundary limit of SCR are considered for classification. The results related to these test events are presented in Table III. From Table III it is evident that if parameters of the kernel are properly tuned, the decision boundary can be established with small number of SVs.

TABLE I

GENERATED ISLANDING AND NONISLANDING EVENTS AT THE PRESENCE OF DIFFERENT TYPES OF LOAD

\begin{tabular}{|c|c|c|c|c|c|c|}
\hline \multirow{3}{*}{$\begin{array}{c}\text { Type of } \\
\text { load }\end{array}$} & \multicolumn{4}{|c|}{ Islanding } & \multirow{2}{*}{\multicolumn{2}{|c|}{$\frac{\text { Nonislanding }}{\text { No. of events }}$}} \\
\hline & \multicolumn{2}{|c|}{ Deficit and excess } & \multicolumn{2}{|c|}{ No. of events } & & \\
\hline & $\Delta \mathrm{P}(\%)$ & $\Delta \mathrm{Q}(\%)$ & Training & Test & Training & Test \\
\hline \multirow{2}{*}{$\begin{array}{c}\text { Const. } \\
P\end{array}$} & $0-45.8$ & $0-50$ & 54 & 276 & \multirow{2}{*}{80} & \multirow{2}{*}{160} \\
\hline & $45.81-100$ & $0-50$ & 26 & 78 & & \\
\hline \multirow{2}{*}{$\begin{array}{c}\text { Const. } \\
I\end{array}$} & $0-58$ & $0-50$ & 60 & 288 & \multirow{2}{*}{80} & \multirow{2}{*}{160} \\
\hline & $58.01-100$ & $0-50$ & 20 & 66 & & \\
\hline \multirow{2}{*}{$\begin{array}{c}\text { Const. } \\
Z \\
\end{array}$} & $0-66$ & $0-50$ & 64 & 296 & \multirow{2}{*}{80} & \multirow{2}{*}{160} \\
\hline & $66.01-100$ & $0-50$ & 16 & 58 & & \\
\hline & Total & & 240 & 1062 & 240 & 480 \\
\hline
\end{tabular}

In order to scrutinize the performance under the most critical islanding cases, the proposed method is further tested with islanding events associated with power imbalance $(\Delta \mathrm{P})$ ranging from $0.5 \%$ to $10 \%$. To accomplish this task, all four scenarios of power imbalance presented in section II-C are taken into account. At first, critical islanding events corresponding to scenario (a), i.e., deficit of active and reactive power imbalance scenario, are tested. A total of 945 islanding events, considering deficit of $\Delta \mathrm{P}$ from $0.5 \%$ to $10 \%$ in conjunction with deficit of $\Delta \mathrm{Q}$ from $0 \%$ to $50 \%$, are generated. These test data 
were a new set and not a subset of the training data. Then detection rate (DR) is obtained using linear, polynomial and Gaussian RBF kernel for each $\Delta \mathrm{P}$ level. Total 480 nonislanding test events as mentioned in Table I are also classified to detect the false alarm (FA) under each $\Delta \mathrm{P}$ level of classification. The results are presented in Table IV and the corresponding performance curve is shown in Fig. 10.

TABLE II

ISLANDING DETECTION USING AN SVM CLASSIFIER WITH LINEAR KERNEL

\begin{tabular}{|c|c|c|c|c|}
\hline \multirow{2}{*}{ Type of load } & \multicolumn{2}{|c|}{ Deficit and excess } & \multirow{2}{*}{$\begin{array}{c}\text { Detection } \\
\text { rate }\end{array}$} & \multirow{2}{*}{$\begin{array}{l}\text { False } \\
\text { alarm }\end{array}$} \\
\hline & $\Delta \mathrm{P}(\%)$ & $\Delta \mathrm{Q}(\%)$ & & \\
\hline \multirow{2}{*}{ Constant $P$} & $0-45.8$ & $0-50$ & $99.64 \%$ & \multirow{2}{*}{$0.63 \%$} \\
\hline & $0-100$ & $0-50$ & $99.72 \%$ & \\
\hline \multirow{2}{*}{ Constant $I$} & $0-58$ & $0-50$ & $100 \%$ & \multirow{2}{*}{$2.5 \%$} \\
\hline & $0-100$ & $0-50$ & $100 \%$ & \\
\hline \multirow{2}{*}{ Constant $Z$} & $0-66$ & $0-50$ & $100 \%$ & \multirow{2}{*}{$2.5 \%$} \\
\hline & $0-100$ & $0-50$ & $100 \%$ & \\
\hline Constant $Z, P$ and $I$ & $0-100$ & $0-50$ & $99.91 \%$ & $1.88 \%$ \\
\hline
\end{tabular}

TABLE III

ISLANDING DETECTION USING SVM CLASSIFIER WITH DIFFERENT KERNELS

\begin{tabular}{|c|c|c|c|c|}
\hline Kernel & Parameter value & SVs & Detection rate & $\begin{array}{c}\text { False } \\
\text { alarm }\end{array}$ \\
\hline Gaussian RBF & $\sigma=1$ & 42 & $97.3 \%$ & $0.4 \%$ \\
\hline Polynomial & $p=2$ & 18 & $98.3 \%$ & $0.2 \%$ \\
\hline
\end{tabular}

From Table IV and Fig. 10, it is observed that under most critical islanding cases, the three kernels show almost similar performance if a trade-off between detection rate and false alarm rate is considered. However, throughout the experimental results and taking less than $1 \%$ false alarm into account, optimistic performance is achieved for polynomial kernel which has successfully classified the islanding events with $\Delta \mathrm{P}$ of $2 \%$ or higher; whereas linear or Gaussian RBF kernel needs the power imbalance of $3 \%$ or higher to achieve $100 \%$ accuracy in classifying the islanding events. Considering the pessimistic performance (with Gaussian RBF or linear kernel) of the proposed method it is revealed that detection rate falls below $100 \%$ when islanding events associated with $\Delta \mathrm{P}<3 \%$ are encountered. However, for $\Delta \mathrm{P} \geq 3 \%$, multifeature-based approach shows satisfactory results even for the pessimistic performance scenarios.

To investigate the classification performance of the proposed method, the other three combinations, scenario (b), (c) and (d) as presented in section II-C, are also taken into account. Thus, a total of 1,440 islanding events are generated considering $\Delta \mathrm{P}$ from $0.5 \%$ to $7 \%$ in conjunction with $\Delta \mathrm{Q}$ from $0 \%$ to $50 \%$ at the presence of three types of loads (constant $Z$, constant $I$ and constant $P$ ). SVM with polynomial kernel of order $3(p=3)$ is applied to test the performance. Test results for scenario (b), (c) and (d) are shown in Table V. From the classification results presented in Table $\mathrm{V}$, it is observed that under two scenarios: (b) deficit of $\Delta \mathrm{P}$ and excess of $\Delta \mathrm{Q}$, and (d) excess of $\Delta \mathrm{P}$ and excess of $\Delta \mathrm{Q}, 100 \%$ accuracy with $0.83 \%$ false alarm is obtained for all the test cases; whereas the scenario (c) represented by the combination of excess of $\Delta \mathrm{P}$ and deficit of $\Delta \mathrm{Q}$ yields $100 \%$ accuracy for $\Delta \mathrm{P} \geq 5 \%$. Thus, the test results prove that proposed method can contribute to the reduction of the size of NDZ of VS relays by detecting islanding events inside SCR for all combinations of deficit and excess of active and reactive power imbalance.

TABLE IV

PERFORMANCE OF SVM ClASSIFIER USING DifFERENT KERNELS UNDER MOST CRITICAL ISLANDING CASES ASSOCIATED WITH DEFICIT OF $\Delta \mathrm{P}$ AND $\Delta \mathrm{Q}$

\begin{tabular}{|c|c|c|c|c|c|c|c|}
\hline \multirow[b]{2}{*}{$\begin{array}{c}\Delta \mathrm{P} \\
(\%)\end{array}$} & \multirow{2}{*}{$\begin{array}{l}\text { No. of } \\
\text { islanding } \\
\text { events }\end{array}$} & \multicolumn{2}{|c|}{ Gaussian RBF } & \multicolumn{2}{|c|}{ Polynomial } & \multicolumn{2}{|c|}{ Linear } \\
\hline & & $\begin{array}{l}\text { DR } \\
(\%)\end{array}$ & $\begin{array}{l}\text { FA } \\
(\%)\end{array}$ & $\begin{array}{l}\text { DR } \\
(\%)\end{array}$ & $\begin{array}{l}\text { FA } \\
(\%)\end{array}$ & $\begin{array}{l}\text { DR } \\
(\%)\end{array}$ & $\begin{array}{l}\text { FA } \\
(\%)\end{array}$ \\
\hline 0.5 & 135 & 89.6 & 0.00 & 92.6 & 0.83 & 82.2 & 0.42 \\
\hline 1 & 135 & 92.6 & 0.00 & 96.3 & 0.83 & 92.6 & 0.42 \\
\hline 2 & 135 & 98.3 & 0.00 & 100 & 0.83 & 99.3 & 0.42 \\
\hline 3 & 135 & 100 & 0.00 & 100 & 0.83 & 100 & 0.42 \\
\hline 5 & 135 & 100 & 0.00 & 100 & 0.83 & 100 & 0.42 \\
\hline 7 & 135 & 100 & 0.00 & 100 & 0.83 & 100 & 0.42 \\
\hline 10 & 135 & 100 & 0.00 & 100 & 0.83 & 100 & 0.42 \\
\hline
\end{tabular}

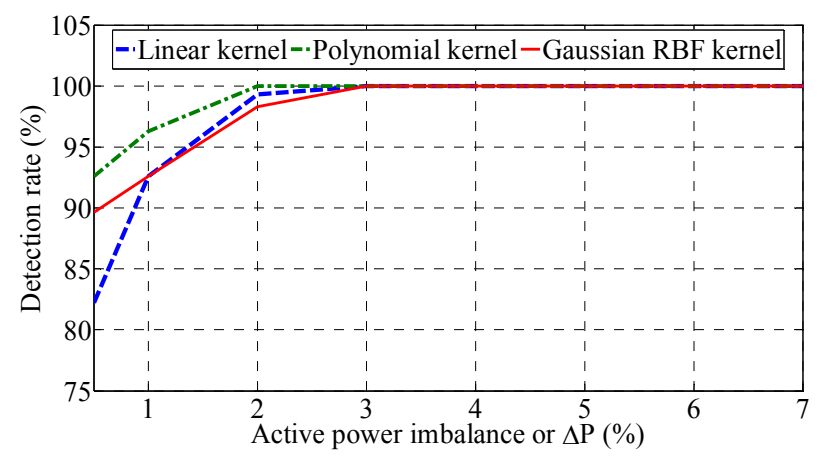

Fig. 10. Performance curve of multifeature-based SVM method with linear, polynomial and Gaussian RBF kernel under most critical islanding cases.

TABLE V

PERFORMANCE OF SVM CLASSIFIER UNDER MOST CRITICAL ISLANDING CASES INCLUDING THREE COMBINATIONS OF DEFICIT AND EXCESS OF $\triangle \mathrm{P}$ AND $\Delta \mathrm{Q}$

\begin{tabular}{|c|c|c|c|c|c|c|c|}
\hline & \multirow{2}{*}{$\begin{array}{c}\Delta \mathrm{P} \\
(\%)\end{array}$} & $\begin{array}{c}\text { No. of } \\
\text { islanding } \\
\text { events }\end{array}$ & $\begin{array}{c}\text { Scenario (c): } \\
\text { Excess } \Delta \mathrm{P} \text { and } \\
\text { Deficit } \Delta \mathrm{Q} \\
(0 \%-50 \%)\end{array}$ & \multicolumn{2}{c|}{$\begin{array}{c}\text { Scenario (b): } \\
\text { Deficit } \Delta \mathrm{P} \text { and } \\
\text { Excess } \Delta \mathrm{Q} \\
(0 \%-50 \%)\end{array}$} & $\begin{array}{c}\text { Scenario (d): } \\
\text { Excess } \Delta \mathrm{P} \text { and } \\
\text { Excess } \Delta \mathrm{Q} \\
(0 \%-50 \%)\end{array}$ \\
\cline { 3 - 9 } & & $\begin{array}{c}\text { DR } \\
(\%)\end{array}$ & $\begin{array}{c}\text { FA } \\
(\%)\end{array}$ & $\begin{array}{c}\text { DR } \\
(\%)\end{array}$ & $\begin{array}{c}\text { FA } \\
(\%)\end{array}$ & $\begin{array}{c}\text { DR } \\
(\%)\end{array}$ & FA (\%) \\
\hline 0.5 & 60 & 91.7 & 0.83 & 100 & 0.83 & 100 & 0.83 \\
\hline 1 & 60 & 91.7 & 0.83 & 100 & 0.83 & 100 & 0.83 \\
\hline 2 & 60 & 91.7 & 0.83 & 100 & 0.83 & 100 & 0.83 \\
\hline 3 & 60 & 91.7 & 0.83 & 100 & 0.83 & 100 & 0.83 \\
\hline 4 & 60 & 91.7 & 0.83 & 100 & 0.83 & 100 & 0.83 \\
\hline 5 & 60 & 100 & 0.83 & 100 & 0.83 & 100 & 0.83 \\
\hline 6 & 60 & 100 & 0.83 & 100 & 0.83 & 100 & 0.83 \\
\hline 7 & 60 & 100 & 0.83 & 100 & 0.83 & 100 & 0.83 \\
\hline
\end{tabular}

Comparative analysis between VS relay and the proposed method is also carried out on the basis of the performance of both methods for islanding detection of DG with $100 \%$ accuracy and with minimum active power imbalance. According to [17], normally VS relay allows the setting of $\alpha$ in the range from $2^{\circ}$ to $20^{\circ}$. Moreover, using the absolute value of $\alpha$ smaller than $2^{\circ}$ may create nuisance tripping and as a result, performance of VS relay may degrade severely. Therefore, in Fig. 11, 100\% accuracy curve of VS relay corresponding to different value of critical active power imbalance $\left(\Delta P_{v s}\right.$ crit $)$ is plotted as a function of absolute value of $\alpha$ considering $|\alpha| \geq$ $2^{\circ}$ and using (6) with equality constraint. This $100 \%$ accuracy 
curve obtained from analytical expression given by (6) is also validated by carrying out repeated dynamic simulation on the test network of Fig. 4 and results are plotted in Fig. 11. From the results presented in Table IV and V, it is observed that for active power imbalance of $5 \%$ or above, the proposed method can provide accurate results and perform effectively, whereas VS relay fails when power imbalance is less than $8.8 \%$ considering the minimum relay setting of $|\alpha|=2^{\circ}$ (as shown in Fig. 11).

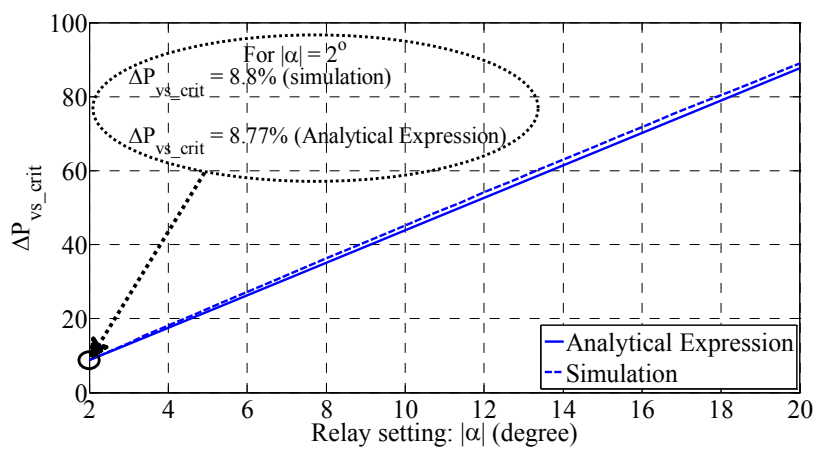

Fig. 11. $100 \%$ accuracy curve of VS relay as a function of $|\alpha|$, on the basis of minimum $\Delta P$ or $\Delta P_{v s_{-} \text {crit }}$ for islanding detection of DG.

As a comparative study with the method given in [2], the proposed method has been tested by simulating the test network of [2]. A total of 54 events have been generated under the same operating conditions and network contingencies as presented in [2]. Thus, five features of the proposed method corresponding to 54 events ( 27 islanding and 27 nonislanding) are generated. Scatter plots of the features are shown in Fig. 12. It should be noted that features of the proposed method were different from the features of [2] and the 27 islanding events represented those islanding cases where $\Delta \mathrm{P} \geq 5 \%$, as reported in [2]. Visual analysis of the proposed features indicates that islanding features can be easily distinguished from the nonislanding features (as shown in Fig. 12). As a result, proposed multifeature-based SVM method shows $100 \%$ accuracy with $0 \%$ false alarm for all test cases. In summary, test results indicate that the proposed technique can detect all islanding events in the test cases, unlike, the method presented in [2], which fails to detect 3 islanding cases at the presence of $5 \%$ power imbalance as reported by the authors.However, the proposed approach is aimed at critical islanding events, which are associated with low $\Delta \mathrm{P}$ (specifically for $\Delta \mathrm{P}<5 \%$ ) in conjunction with all possible combinations (deficit and excess) of active and reactive power imbalance. For those cases, the two classes are not linearly separable, as shown in Fig. 13. Using the proposed approach, those critical islanding events can be easily classified as demonstrated using the test network earlier (see Tables IV and V).
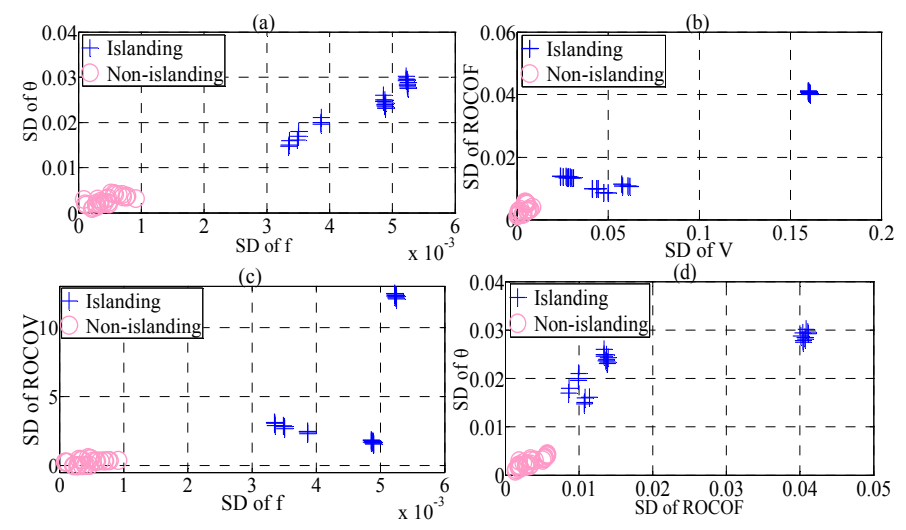

Fig. 12. Scatter plots of features for 54 events of [2]: (a) Standard Deviation (SD) of $\theta$ versus SD of $f$, (b) SD of ROCOF versus SD of $V$, (c) SD of ROCOV versus SD of $f$, (d) SD of $\theta$ versus SD of ROCOF.
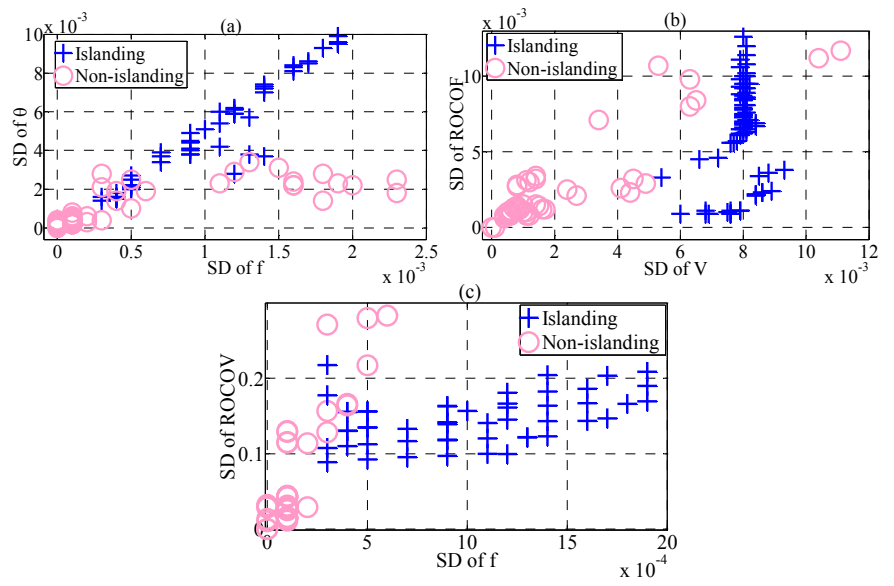

Fig. 13. Scatter plots of features for nonislanding and islanding events with $\Delta \mathrm{P}<5 \%$ : (a) SD of $\theta$ versus SD of $f$, (b) SD of ROCOF versus SD of $V$, (c) SD of ROCOV versus SD of $f$.

Proposed multifeature-based approach can be applied in real networks provided that the SVM is trained with most expected circuit configurations and covering all forms of power imbalance level for islanding events along with all possible types of nonislanding events which may occur in the system. Moreover, the speed of response of the algorithm embedded relay is expected to be fast, given the fact that the features extracted in real-time are passed through the trained SVM containing a small number of support vectors. Since the features of the proposed approach depend only on the terminal voltage parameters (amplitude, frequency, phase angle, etc.), retraining is not needed for this algorithm embedded relay, if minor changes occur in the system, e.g., if a new line or DG is installed, or a normally open point is shifted. To confirm this assertion, the trained SVM, which was kept unchanged, was tested with several test cases (A total of 640 islanding events) generated separately by eliminating one branch (DL-3), two branches (DL-3 and the branch connected through Dxfm-2) and shifting the open point (near the bus bar in between DL-1 and DL-2, and near the bus bar in between DL-2 and DL-3) in the test network of Fig. 9. The test results showed almost similar performance (performance deteriorates by only $1 \%$ in DR) as presented in Tables II and III. Numerically, in the proposed approach, active power imbalance of $0 \%$ to $100 \%$ and reactive 
power imbalance of $0 \%$ to $50 \%$ in the islanded system, were considered by varying the load and generation profiles (applying the procedure presented in [16]). For islanding cases, variations of active and reactive power imbalance, play a vital role in the variation of system parameters such as voltage, frequency, phase angle, etc. In the proposed approach, all possible combinations of active and reactive power imbalances were taken into account. Therefore, the trained SVM classifier is expected to be independent of network topology. Nevertheless, minor changes in the network can decrease the accuracy slightly ( $1 \%$ is obtained in the above test). But, this slight degradation of accuracy may be avoided, if larger volume of training data is considered.

Therefore, retraining is not required if minor changes occur in the system provided that SVM is trained with larger volume of data covering all possible combinations of islanding and nonislanding events. However, for safe operation of the network with the distributed generation, if significant changes in network topology take place, for example, addition or disconnection of large DG unit and addition or disconnection of line that plays a significant role in the operation of the network, then the system must be modelled at first to generate the training data and the SVM has to be retrained accordingly.

\section{CONCLUSION}

This paper presents a multifeature-based SVM classification technique to detect islanding, especially under critical islanding cases where VS relays fail to trigger. Several islanding and nonislanding events have been generated by simulating the practical scenarios in a test network. Using the tuned parameters of trained SVM, pessimistic islanding cases are tested and detected. Test results indicate that the proposed method is able to detect islanding inside the subcritical region, which corresponds to a sub-region of nondetection zone (NDZ) of VS relays. The contributions of this paper can be summarized as follows:

- Boundary limit of subcritical region (SCR) associated with VS relays is established, and investigated at the presence of constant $Z$, constant $I$ and constant $P$ load.

- Islanding events at the presence of constant $Z$, constant $I$ and constant $P$ load along with all possible combinations of deficit and excess of active and reactive power imbalance, are detected by the proposed method with a high degree of accuracy, and the rate of false alarm is limited.

- Proposed method is scrutinized by testing several critical islanding events associated with low active power imbalance (for both, deficit and excess) in combinations with deficit and excess of reactive power imbalance.

- For the example system, the proposed technique has successfully detected the islanding events for active power imbalance of $5 \%$ and above; whereas VS relay, with minimum setting value of $2^{\circ}$, fails when power imbalance is less than $8.8 \%$.

\section{REFERENCES}

[1] S.K. Salman, "The impact of embedded generation on voltage regulation and losses of distribution networks", in Proc. The Impact of Embedded Generation on Distribution Networks (Digest No. 1996/194), IEE Colloquium, London, pp. 2/1-2/5, 15 Oct. 1996.
[2] K. El-Arroudi, G. Joos, I. Kamwa, and D. T. McGillis, "Intelligent-based approach to islanding detection in distributed generation," IEEE Trans. Power Del., vol. 22, no. 2, pp. 828-835, Apr. 2007.

[3] Faqhruldin, O.N., El-Saadany, E.F. and Zeineldin, H.H., "Evaluation of islanding detection techniques for inverter-based distributed generation," in Proc. 2012 IEEE PES General Meeting, San Diego, July 2012.

[4] H. H. Zeineldin and M. M. A. Salama, "Impact of load frequency dependence on the NDZ and performance of the SFS islanding detection method," IEEE Trans. Ind. Electronics, vol. 58, no. 1, pp. 139-146, Jan. 2011.

[5] A. Yafaoui, B. Wu, S. Kouro, "Improved Active Frequency Drift Antiislanding Detection Method for Grid Connected Photovoltaic Systems," IEEE Trans. Power Electronics, vol. 27, no. 5, pp. 2367-2375, May 2012.

[6] H. Karimi, A. Yazdani, R. Iravani, "Negative-Sequence Current Injection for Fast Islanding Detection of a Distributed Resource Unit," IEEE Trans. Power Electronics, vol. 23, no. 1, pp. 298-307, Jan. 2008.

[7] W. Freitas, Wilsun Xu, C. M. Affonso, and Zhenyu Huang, "Comparative analysis between ROCOF and vector surge relays for distributed generation applications," IEEE Trans. Power Del., vol. 20, no. 21, pp. 1315-1324, Apr. 2005.

[8] K. El-Arroudi, and G. Joos, "Data mining approach to threshold settings of islanding relays in distributed generation," IEEE Trans. Power Del., vol. 22, no. 3, pp. 1112-1119, Aug. 2007.

[9] S. R. Samantaray, K. El-Arroudi, G. Joos, and I. Kamwa, "A fuzzy rulebased approach for islanding detection in distributed generation," IEEE Trans. Power Del., vol. 25, no. 3, pp. 1427-1433, Jul. 2010.

[10] M. S. Thomas, and P. P. Terang, "Islanding detection using decision tree approach," in Proc. Joint Int. Conf. on Power Electronics, Drives and Energy Systems (PEDES) and 2010 Power India, New Delhi, Dec. 2023, 2010.

[11] A. Pigazo, M. Liserre, R. A. Mastromauro, V. M. Moreno, and A. Dell'Aquila, "Wavelet-based islanding detection in grid-connected PV systems," IEEE Trans. Industrial Electronics, vol. 56, no. 11, pp. 44454455, Nov. 2009.

[12] V. N. Vapnik, Statistical Learning Theory. New York: Wiley, 1998.

[13] N. Cristianini, and J. Shawe-Taylor, An Introduction to Support Vector Machines and Other Kernel-based Learning Methods. Cambridge, MA: Cambridge Univ. Press, 2000.

[14] Peter G. V. Axelberg, I.Y.-H. Gu, and M. H. J. Bollen, "Support vector machine for classification of voltage disturbances," IEEE Trans. Power Del., vol. 22, no. 3, pp. 1297-1303, Jul. 2007.

[15] Faqhruldin, O.N, "A Universal Islanding Detection Technique for Distributed Generation Using Pattern Recognition,” M.Sc. Thesis, University of Waterloo, Waterloo, Ontario, Canada, 2013.

[16] Jose C. M. Vieira, Walmir Freitas, Wilsun Xu, and Andre Morelato, "An investigation on the nondetection zones of synchronous distributed generation anti-islanding protection," IEEE Trans. Power Del., vol. 23, no. 2, pp. 593-600, Apr. 2008.

[17] Walmir Freitas, Zhenyu Huang, and Wilsun Xu, "A practical method for assessing the effectiveness of vector surge relays for distributed generation applications," IEEE Trans. Power Del., vol. 20, no. 1, pp. 5763, Jan. 2005.

[18] C. L. Wadhwa, Electrical Power Systems. Wiley Eastern Ltd., $4^{\text {th }}$ Edition, 2006.

[19] Shigeo Abe, Support Vector Machines for Pattern Classification. London: Springer, 2005.

\section{BIOGRAPHIES}

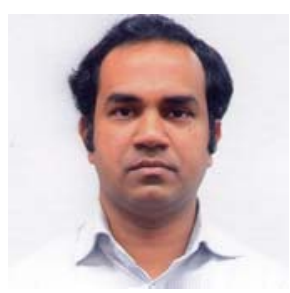

Mollah Rezaul Alam (Std. Member'12) received the B.Sc. degree in Electrical and Electronic Engineering from Bangladesh University of Engineering \& Technology (BUET), Dhaka, Bangladesh in 2005. Currently, he is pursuing the $\mathrm{Ph} . \mathrm{D}$. degree in electrical engineering at the University of Wollongong, New South Wales, Australia.

Prior to starting Ph.D. studies, he was involved in the telecommunication industry in Bangladesh for 5 years, where he worked in the area of Intelligent Network \& Value Added Services of cellular mobile technology. His research interests include computational intelligence, data 
mining, fault detection, classification and analysis considering the impacts of distributed energy resources.

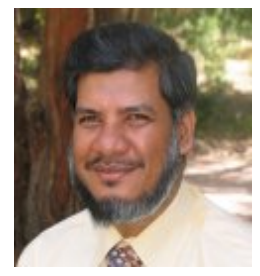

Kashem M. Muttaqi (M'01, SM'05) received the B.Sc. degree in electrical and electronic engineering from Bangladesh University of Engineering and Technology (BUET), Bangladesh in 1993, the M.Eng.Sc. degree in electrical engineering from University of Malaya, Malaysia in 1996 and the Ph.D. degree in Electrical Engineering from Multimedia University, Malaysia in 2001. Currently, he is an Associate Professor at the School of Electrical, Computer, and Telecommunications Engineering, and member of Australian Power Quality and Reliability (APQRC) at the University of Wollongong, Australia. He was associated with the University of Tasmania, Australia as a Research Fellow/Lecturer/Senior Lecturer from 2002 to 2007, and with the Queensland University of Technology, Australia as a Research Fellow from 2000 to 2002. Previously, he also worked for Multimedia University as a Lecturer for three years. He has more than 17 years of academic experience and has authored or coauthored over 165 papers in international journals and conference proceedings. His research interests include distributed generation, renewable energy, electrical vehicles, smart-grid, power system planning and control. Dr. Muttaqi is an Associate Editor of the IEEE TRANSACTIONS ON INDUSTRY APPLICATIONS.

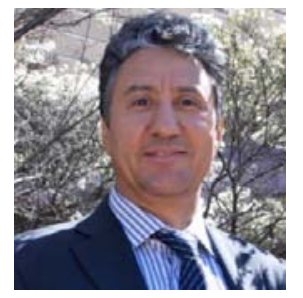

Abdesselam Bouzerdoum (M'89-SM'03) received the M.Sc. and Ph.D. degrees in electrical engineering from the University of Washington, Seattle.

In 1991, he joined The University of Adelaide, Adelaide, Australia, and in 1998, he was appointed an Associate Professor with Edith Cowan University, Perth, Australia. Since 2004, he has been with the University of Wollongong, Wollongong, Australia, as Professor of Computer Engineering, where he also served as Head of School of Electrical, Computer and Telecommunications Engineering from 2004 to 2006 and Associate Dean (Research), with the Faculty of Informatics, from 2007 to 2013. From 2009 to 2011, he was a Member of the Australian Research Council College of Experts, and served as Deputy Chair of the Engineering, Mathematics and Informatics panel from 2010 to 2011.

Dr. Bouzerdoum was the recipient of numerous awards and prizes; the most notable are the Eureka Prize for Outstanding Science in Support of Defence or National Security in 2011, the Chester Sall Award in 2005, and a Distinguished Researcher Award (Chercheur de Haut Niveau) from the French Ministry of Research in 2001. He has published over 280 technical articles and graduated many Ph.D. and Masters students. From 1999 to 2006, he served as an Associate Editor of IEEE TRANSACTIONS ON SYSTEMS, MAN, AND CYBERNETICS. 